\title{
Effectiveness of the Sleep Enhancement by Green Romaine Lettuce (Lactuca sativa) in a Rodent Model
}

\author{
Hyo-Won Kim, ${ }^{a}$ Hyung Joo Suh, ${ }^{b}$ Hyeon-Son Choi, ${ }^{c}$ Ki-Bae Hong, ${ }^{d}$ and Kyungae Jo*,b \\ ${ }^{a}$ Department of Biotechnology, Graduate School, Korea University; Seoul 02841, Republic of Korea: ${ }^{b}$ Department \\ of Public Health Science, Korea University; Seoul 02841, Republic of Korea: ${ }^{c}$ Department of Food Science and \\ Technology, Seoul Women's University; Seoul 01797, Republic of Korea: and ${ }^{d}$ BK21Plus, College of Health Science, \\ Korea University; Seoul 02841, Republic of Korea. \\ Received May 30, 2019; accepted July 24, 2019
}

This study was conducted to investigate the effects of the extracts of green romaine lettuce (GRE) on sleep enhancement. GRE contains 1071.7 and $199.2 \mu \mathrm{g} / \mathrm{g}$ of extracts of lactucin and lactucopicrin, respectively, known as sleep enhancement substances. When $100 \mathrm{mg} / \mathrm{kg}$ of GRE was administered orally, sleep latency and duration time were significantly increased compared to controls $(p<0.05)$. Rapid eye movement (REM) sleep decreased with $100 \mathrm{mg} / \mathrm{kg}$ of GRE administration and non-REM (NREM) sleep also increased. There was no significant difference between REM and NREM among the oral GRE administration groups receiving 100,120 , and $160 \mathrm{mg} / \mathrm{kg}$ GRE. In the caffeine-induced insomnia model, total sleep time was significantly increased by $100 \mathrm{mg} / \mathrm{kg}$ GRE administration compared to the caffeine-treated group $(p<0.05)$. In addition, GRE inhibited the binding of $\left[{ }^{3} \mathrm{H}\right]$-flumazenil in a concentration-dependent manner, and affinity of both lactucin and lactucopicrin to gamma-aminobutyric acid $(\mathrm{GABA})_{\mathrm{A}}$-benzodiazepine $(\mathrm{BDZ})$ receptor was $80.7 \%$ and $55.9 \%$, respectively. Finally, in the pentobarbital-induced sleep mouse model, the sleep enhancement effect of GRE was inhibited by flumazenil, an antagonist of BDZ. Thus, these results demonstrate that GRE acts via a GABAergic mechanism to promote sleep in a rodent model.

Key words Lactuca sativa; sleep; lactone; pentobarbital; electroencephalogram; rodent model

\section{INTRODUCTION}

Lactuca sativa (lettuce) is a type of lettuce with sturdy, crunchy, and dark green leaves. It is also known as cos or romaine lettuce in North America. ${ }^{1)}$ Romaine lettuce is used as a savory salad with high fiber and low calories. ${ }^{2)}$ It has traditionally been used in the treatment of insomnia, anxiety, neurosis, dry coughs, and stomach problems. ${ }^{1,3)}$ In particular, the leaves of this plant have been known to promote anticonvulsant and sedative-hypnotic effects and the seeds have been studied in analgesic and anti-inflammatory activity in rats. ${ }^{4,5}$

Sleep is a crucial physiological state to maintain normal body function. In particular, sleep has important effects on mental and physical health, promotes brain activity in learning and memory, and supports immune function and hormonal balance. ${ }^{6)}$ Sleep proceeds in four stages that include rapid eye movement (REM) and non-rapid eye movement (NREM). REM sleep is also called paradoxical sleep because brain waves demonstrate an awake pattern. In NREM sleep, the delta wavelength is indicated and deep sleep is attained. ${ }^{7)}$ During sleep, these stages are repeated 4-5 times in a cycle and all stages are indicated by the different brain waves. Moreover, rodents show the same sleep pattern as humans; thus sleep architecture analysis through an animal model is possible. $^{8)}$

Insufficient sleep is one factor causing various mental and physical problems. A lack of sleep has been known to lead to difficulties in daily life, including lethargy and memory failure, which are accompanied by traffic and work-related accidents. ${ }^{9}$ A chronic lack of sleep is involved in the occurrence of various diseases. Illnesses such as cardiovascular disease, diabetes, obesity, and depression are reported to be linked to insomnia, a sleep disorder. ${ }^{10)}$ The number of people experiencing insomnia is continually increasing worldwide; approximately one-third of the population are struggling with a sleep disorder. ${ }^{10)}$

The treatment of insomnia has been performed using pharmacological agents such as benzodiazepine (BDZ). However, sustained use of this drug could cause side effects, including poor job performance, drug resistance/addiction, and depression. ${ }^{11)}$ Recently, nonpharmacologic methods using natural plants have been observed or suggested as an alternative approach with milder adverse effects. Several herbs have been reported to have a sedative or sleep-promoting capacity: valerian (Valeriana officinalis), hops (Humulus lupulus), German chamomile (Matricaria recutita), passionflower (Passiflora incarnata), and Polygonatum sibiricum. ${ }^{12-15)}$ Our previous study revealed that the mixture of Lactuca sativa and skullcap extracts played an active role in sleep promotion. ${ }^{16)}$ In this study, we focus on the sleep-promoting effect of romaine lettuce and its mechanisms by investigating antagonistic behaviors on sleep-related receptors.

\section{MATERIALS AND METHODS}

Preparation of Extract Green romaine lettuce leaves were purchase from the local market. Fresh leaves of lettuce were washed with water, dried at $60^{\circ} \mathrm{C}$, milled, and stored in the refrigerator. Lettuce leaf powder $(100 \mathrm{~g})$ was extracted three times with $70 \%(\mathrm{v} / \mathrm{v})$ ethanol $(500 \mathrm{~mL})$ for $4 \mathrm{~h}$ in a Soxhlet apparatus. The combined ethanol extracts were concentrated to $25 \%$ of total volume of the extracts using a rotary 


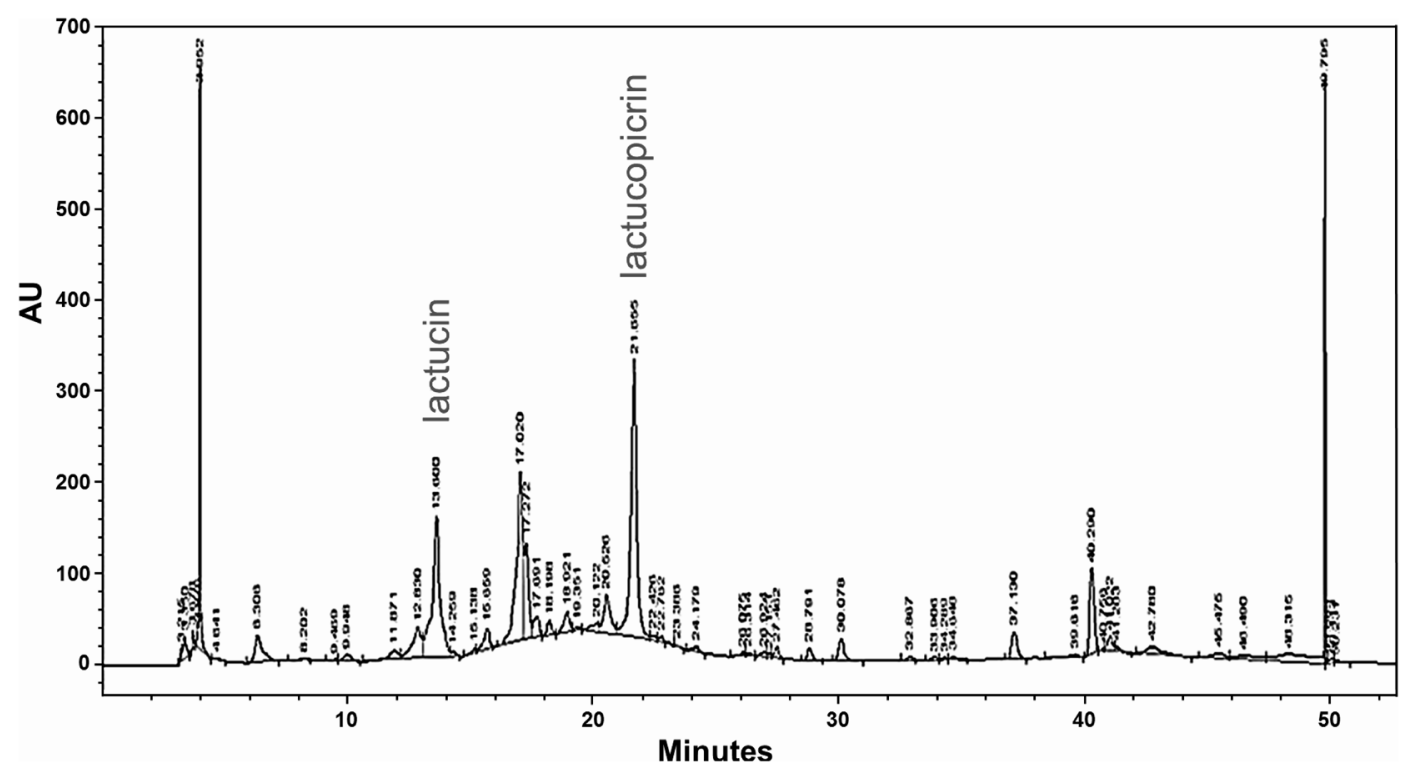

Fig. 1. HPLC Chromatogram for Lactucin and Lactucopicrin in Green Romaine Leaf Extract (GRE)

evaporator. The concentrates were lyophilized for further experiments.

Assay of Lactucin and Lactucopicrin The sesquiterpene lactones from green romaine lettuce extract (GRE) were analyzed by HPLC system (Agilent, Waldbronn, Germany) according to the method of Abu-Reidah et al. ${ }^{17)}$ A C18 column $(4.6 \times 150 \mathrm{~mm}, 5 \mu \mathrm{m}$, Phenomenex, U.S.A.) was employed, using the following elution: $0 \mathrm{~min}, 85 \% \mathrm{~A} ; 5 \mathrm{~min}, 85 \% \mathrm{~A}$; $35 \mathrm{~min}, 100 \% \mathrm{~B}$; $45 \mathrm{~min}, 100 \% \mathrm{~B}$; $46 \mathrm{~min}, 85 \% \mathrm{~A} ; 60 \mathrm{~min}$, $85 \% \mathrm{~A}$; solvents: $\mathrm{A}=$ phosphoric acid $0.2 \%(\mathrm{v} / \mathrm{v}), \mathrm{B}=$ acetonitrile. The flow rate was $0.7 \mathrm{~mL} / \mathrm{min}$, lactones were identified at a wavelength of 254 and $320 \mathrm{~nm}$, and the injection quantity was $20 \mu \mathrm{L}$.

Experimental Animals All animals (ICR mice and Sprague-Dawley (SD) rats) were obtained from Central Lab. Animal Inc. (Seoul, Korea). During the experimental period, water and diet were freely provided at a room temperature of $24^{\circ} \mathrm{C}$ and a relative humidity of $50-60 \%$ under a 12-h light/dark cycle. After a week-long adaptation period, pentobarbital-induced sleep tests were performed using mice and the rats were used for electroencephalogram (EEG) measurements. The animal experiments were reviewed and approved by the Korea University Animal Care Committee (KUIACUC-2015-286).

Pentobarbital-Induced Sleep Test Mice were starved without feeding for $24 \mathrm{~h}$ and pentobarbital $(42 \mathrm{mg} / \mathrm{kg}$ ) was intraperitoneally injected after $40 \mathrm{~min}$ of oral administration of $\operatorname{GRE}(80,100,120$, and $160 \mathrm{mg} / \mathrm{kg})$ in $0.9 \%$ physiological saline. Meanwhile, in order to investigate whether GRE affects sleep in mice with receptor blockade through the use of antagonist, flumazenil $(3.5 \mathrm{mg} / \mathrm{kg}$, Sigma-Aldrich Inc., St. Louis, MO, U.S.A.) was intraperitoneally injected $30 \mathrm{~min}$ before oral administration of BDZ $(2.5 \mathrm{mg} / \mathrm{kg}$ ) or GRE (80 and $100 \mathrm{mg} / \mathrm{kg}$ ). Animals that did not fall asleep within $15 \mathrm{~min}$ were excluded from the experiment and sleep duration was defined as the time to recover from the instant the clinging reflexes disappeared. Individual treatments were not revealed to the observes and sleep latency and sleep duration were measured according to previously reported methods. ${ }^{18)}$

EEG Analysis The rats were anesthetized with $2 \%$ isoflu- rane (Troikaa Pharmaceutical Ltd., Gujarat, India) and secured to the stereotaxic instrument frame (Stoelting Inc., Wood Dale, IL, U.S.A.). After exposing the skull by dissecting the scalp and releasing the fascia, four holes were drilled to insert the electrodes into the surface of the skull corresponding to the frontal cortex, striatum, and hippocampus, and the screws and electrodes were fixed with dental cement.

After the operation, antibiotics were administered and the rats recuperated in individual cages for $7 \mathrm{~d}$ and were then divided into the control and the treatment groups. After oral administration of $\operatorname{GRE}(80,100,120$, and $160 \mathrm{mg} / \mathrm{kg}$ ), Iox2 (version 2.8.0.13, Emka Technologies, Paris, France) was used from 10:00 am to 5:30 pm for brain wave recording over $9 \mathrm{~d}$. EEG spectra were analyzed at $1-\mathrm{Hz}$ bin and the standard frequency domain ( $\gamma$ wave: $30-60 \mathrm{~Hz}, \beta$ wave: 12-30, $\alpha$ wave: $9-12, \theta$ wave: $4-9 \mathrm{~Hz}, \delta$ wave: $0.5-4 \mathrm{~Hz}$ ) were examined. After recording, Fast Fourier transform (FFT) data were collected at intervals of $2 \mathrm{~s}$ and the wake time and sleep time were calculated using the ecgAUTO3 program (version 3.3.0.20, Emka Technologies) with an average value of FTT data ranging from 0 to $30 \mathrm{~Hz}$ for every $10 \mathrm{~s}$.

In the caffeine-induced model, EEG analysis was performed for $4 \mathrm{~d}$ and GRE and caffeine $(15 \mathrm{mg} / \mathrm{kg})$ were orally administered before EEG recording. Other experimental processes were the same for EEG acquisition and analysis.

Gamma-Aminobutyric Acid (GABA) $)_{A}-B D Z$ Receptor Binding Assay A GABA $A_{A}-B D Z$ receptor binding assay was performed according to the method previously described by Risa et al. ${ }^{19)}$ with some slight modifications. Cerebral cortical membranes of male SD rats were prepared in cold Tris- $\mathrm{HCl}$ buffer $\left(30 \mathrm{mM}, \mathrm{pH}\right.$ 7.4) following a previous report. ${ }^{15)}$ For membrane-binding experiments, $33.3 \mu \mathrm{g}$ membrane protein $(100 \mu \mathrm{L})$ was added to $25 \mu \mathrm{L}$ samples (GRE, lactucin, and lactucopicrin) and $21 \mu \mathrm{L}\left[{ }^{3} \mathrm{H}\right]$-flumazenil $(0.8 \mathrm{nM}$, final concentration in assay), mixed, and incubated on ice for $40 \mathrm{~min}$. Total binding and nonspecific binding of $\left[{ }^{3} \mathrm{H}\right]$-flumazenil were estimated in the presence of the binding buffer and BDZ $(1 \mu \mathrm{M})$, respectively. After incubation, the mixture was poured directly onto glass fiber filters (Brandel Inc., Gaithersburg, MD, U.S.A.) under suction and immediately washed 3 times 
with ice-cold buffer. Radioligands bound to the membrane were counted by a Hidex 300SL counter (Hidex, Finland). For all receptor binding assays, the binding displacement was calculated as follows:

$$
\begin{aligned}
& \text { Binding displacement }(\%) \\
& \quad=\left[1-\left(\mathrm{DPM}_{\mathrm{GRE}}-\mathrm{DPM}_{\mathrm{NSB}}\right) /\left(\mathrm{DPM}_{\mathrm{TB}}-\mathrm{DPM}_{\mathrm{NSB}}\right)\right] \times 100
\end{aligned}
$$

(DPM: disintegrations per minute, TB: total binding, and NSB: nonspecific binding).

Statistical Analysis Experimental results were expressed as means \pm standard error of the mean (S.E.M.). The statistical significance for one-way or two-way ANOVA, followed by post-hoc Tukey multiple comparison test, was set at $p<0.05$ using the Statistical Package for Social Sciences version 12.0 (SPSS Inc., Chicago IL, U.S.A.).

\section{RESULTS}

Analysis of Lactucin and Lactucopicrin in GRE The content of these bitter compounds was analyzed using HPLC system (Fig. 1). Lactucin and lactucopicrin contents were 1071.7 and $199.2 \mu \mathrm{g} / \mathrm{g}$ of extract, respectively (data not shown). In our previous study, ${ }^{20)}$ the extraction yield of romaine leaf was reported as $37.1 \%$.

Effect of GRE on Sleep Latency and Total Sleep Duration in the Pentobarbital-Induced Sleep of Mice The effects of GRE on sleep latency and duration were investigated in a pentobarbital-induced model (Fig. 2). The sleep latency of pentobarbital-induced mice was not significantly different in the green romaine leaf extract-treated groups compared to the normal group (Fig. 2A). On the other hand, the mice that received a $100 \mathrm{mg} / \mathrm{kg}$ dose showed the longest sleep duration ( $80 \mathrm{~min}$ ) and the increased duration showed a decreasing trend with dosage, although statistical significance was not observed (Fig. 2). However, dosages more than $100 \mathrm{mg} / \mathrm{kg}$ were still shown to be effective in the increase of sleep duration compared with normals. This result indicates that GRE at $100 \mathrm{mg} / \mathrm{kg}$ can facilitate the action of pentobarbital-inducing sleep by shortening latency, resulting in extended sleep duration.

Effects of GRE on Sleep Architecture To investigate

A

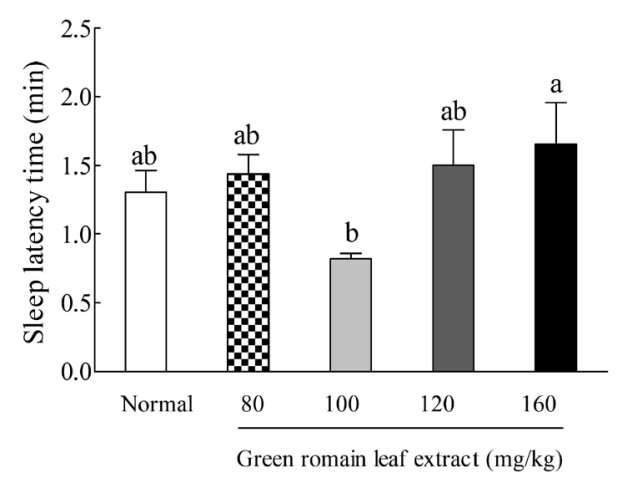

the effect of GRE on sleep quality and quantity, sleep architectures were analyzed using EEG for rats receiving green romaine lettuce (Fig. 3). The GRE-administration groups (100, 120 , and $160 \mathrm{mg} / \mathrm{kg}$ ) demonstrated significant increases in total sleep times (Fig. 3E) $(p<0.05)$. In particular, a dose of $100 \mathrm{mg} / \mathrm{kg}$ increased sleep time by approximately $14 \%$ compared with the normal group. In contrast, wake time, which decreased with GRE administration, was negatively correlated to total sleep time (Fig. 3F). In particular, GRE (100, 120, and $160 \mathrm{mg} / \mathrm{kg}$ ) increased the duration of delta wave by about 2.1 times (approximately 3.6h) compared with control group (Fig. $3 \mathrm{~B})$. However, the theta wave, which means relatively shallower than delta wave, showed no significant difference in GRE treatments compared to the normal group (Fig. 3C). This result showed that GRE improves the sleep quality by increasing deep sleep. Although GRE administration of $100 \mathrm{mg} / \mathrm{kg}$ or more showed similar effects on sleep time and sleep quality (Fig. 3), sleep latency time for the $100 \mathrm{mg} / \mathrm{kg}$ dosage was superior to the 120 or $160 \mathrm{mg} / \mathrm{kg}$ dosages. Therefore, GRE administration at $100 \mathrm{mg} / \mathrm{kg}$ may be effective in improving sleep. This increase in sleep time resulting from GRE was involved in a change of sleep patterns. NREM sleep time increased with GRE administration, while REM decreased (Figs. 3A, D). In particular, $120 \mathrm{mg} / \mathrm{kg}$ of GRE increased NREM by approximately $67 \%$ compared with normals, while REM sleep time decreased by $50 \%$ with the same dosage. Thus, GRE improved total sleep time and quality by increasing NREM, and increased NREM sleep was found to be due to increased delta wave time (Figs. 3A, B).

Effect of GRE on Sleep Architecture in a CaffeineInduced Wake Model Sleep architectures of the green romaine lettuce administration in a caffeine-induced awake model are presented in Fig. 4. GRE dosages of $100 \mathrm{mg} / \mathrm{kg}$, which is considered effective in sleep latency and sleep quality assessment, and $80 \mathrm{mg} / \mathrm{kg}$, slightly lower, were administered orally. Caffeine led to a significant decreases in sleep time and NREM compared with the normal group; in contrast, wake time increased with caffeine administration (Fig. 4). However, GRE co-treatment reversed the effect of caffeine on sleep architecture. NREM increased with the co-treatment of GRE and caffeine, but statistical significance was not observed. Sleep time significantly increased (approximately 15\%)

\section{B}

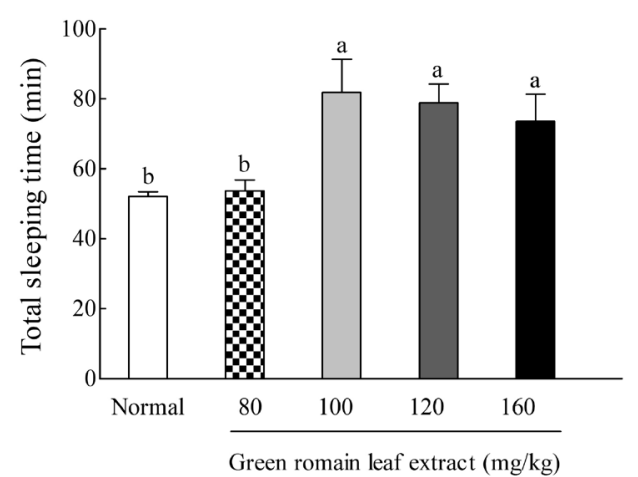

Fig. 2. Effects of Administration of Green Romaine Leaf Extracts (GRE) by Dosage on Sleep Latency Time (A) and Sleep Duration (B) in Mice Intraperitoneal Injected a Hypnotic Dosage of Pentobarbital (42 mg/kg, i.p.)

Mice received pentobarbital $40 \mathrm{~min}$ after oral administration of green romaine leaf extracts by dosage. The normal group was administered only $0.9 \%$ physiological saline. Each column represents the mean \pm standard error of the mean (S.E.M.) calculated from 8 mice. Different letters indicate significant differences $(p<0.05)$ among samples by one-way ANOVA followed by post-hoc Tukey test. 


\section{A. NREM}

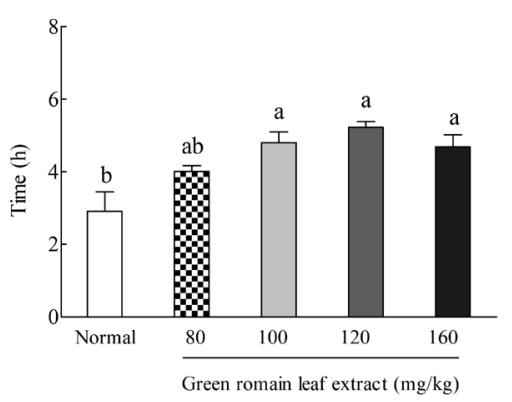

D. REM

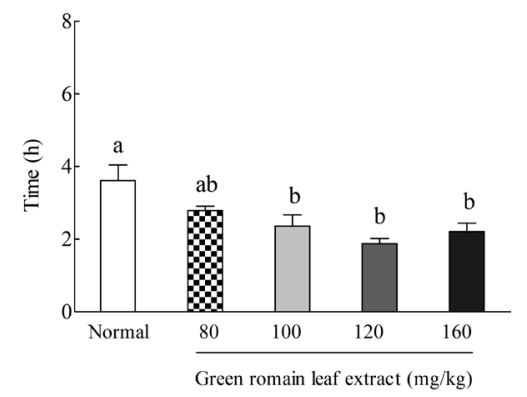

B. $\delta$ wave

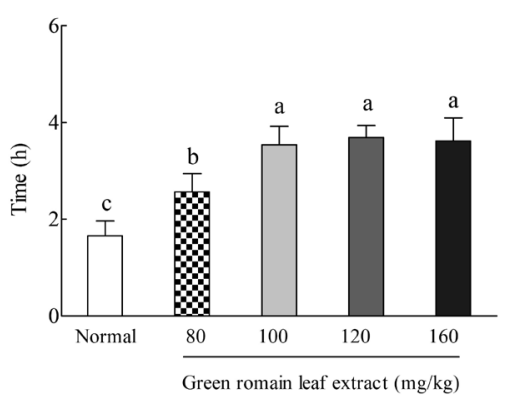

E. Sleep

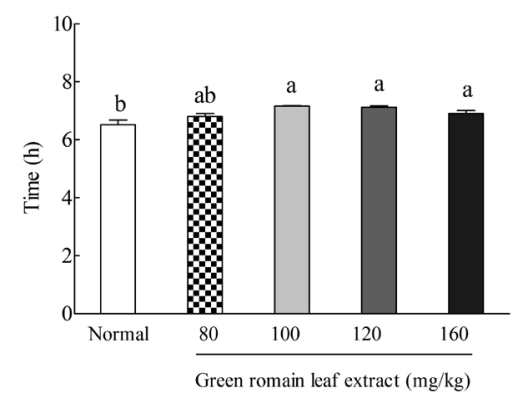

C. $\theta$ wave

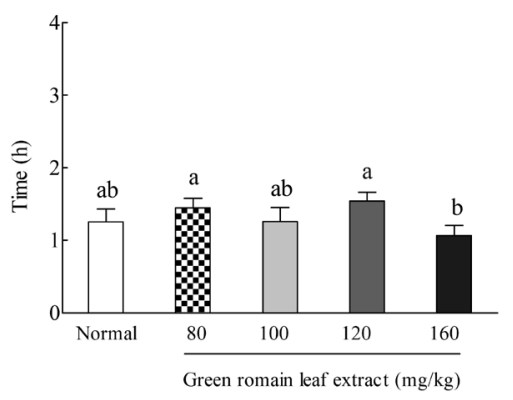

F. Awake

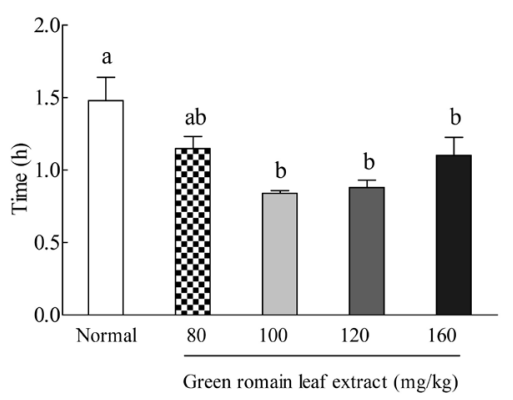

Fig. 3. Effects of Administration of Green Romaine Lettuce Leaf Extracts (GRE) by Dosage on Sleep Quality and Quantity

Values are the mean \pm S.E.M. calculated from 6 rats. The normal group was administered only $0.9 \%$ physiological saline. Different letters indicate significant differences $(p<0.05)$ among samples by one-way ANOVA followed by post-hoc Tukey test.

with $100 \mathrm{mg} / \mathrm{kg}$ of GRE, attaining a normal level. GRE cotreatment decreased wake time by approximately $35 \%$ compared with caffeine-only controls (Fig. 4D). However, REM was further increased with GRE co-treatment compared with caffeine-only controls.

GABA $_{A}$-Benzodiazepine Receptor-Binding Activity of GRE and Active Compounds Figure 5A shows the GREmediated displacement of $\left[{ }^{3} \mathrm{H}\right]$-flumazenil binding (\%) found in the present study. GRE significantly increased displacement of $\left.{ }^{3} \mathrm{H}\right]$-flumazenil from 11.9 to $81.6 \%$ in a concentrationdependent manner $(p<0.05)$. The expected lactucin and lactucopicrin as sleep-promoting active ingredients of GRE were exhibited binding activity of $80.7 \%$ and $55.9 \%$, respectively, which resulted on significantly higher binding activity $(p<0.05)$. These results suggest that lactucin and lactucopicrin, which are contained in GRE, bind effectively to $\mathrm{GABA}_{\mathrm{A}}$ receptor and are an active substance that promotes sleep.

Effects of GABAergic Antagonists on GRE Mediated Sleep Behavior in a Pentobarbital-Induced Sleep Mouse Model At a hypnotic dose of pentobarbital $(42 \mathrm{mg} / \mathrm{kg}$, intraperitoneal (i.p.) injection) (Fig. 6), GRE (100 $\mathrm{mg} / \mathrm{kg}$ ) and BDZ significantly decreased the sleep latency, while flumazenil $\left(\mathrm{GABA}_{\mathrm{A}}-\mathrm{BDZ}\right.$ receptor antagonist) reversed this results. Flumazenil increased GRE-mediated sleep latency by $37 \%$ compared to that in the not treated with flumazenil group (Fig. 6A). In addition, total sleeping time was significantly increased with GRE treatment in a dose-dependent manner. However, flumazenil decreased this GRE-mediated sleeping duration by $14 \%$ (Fig. 6B). This result demonstrates that flumazenil eliminates a GRE-mediated sleep promotion effect in vivo.

\section{DISCUSSION}

Lettuce has been known to contain hypnotic substances that induce sleep. Those components include lactucin and lactucopicrin, which impart a bitter taste. ${ }^{20)}$ Romaine lettuce contains a variety of flavonoids including lactucin and lactucopicrin. Previous studies have reported contents of caftaric acid, chlorogenic acid, chicoric acid, and isochlorogenic acid as $1.9,1.2,3.9$, and $0.3 \mathrm{mg} / \mathrm{g}$ of extract, respectively. ${ }^{21)} \mathrm{The}$ major constituents found in lettuce species are sesquiterpene lactones, such as lactucin, lactucopicrin, and their derivatives. The sesquiterpene lactone derivatives have been reported to promote various physiological activities such as cytotoxicity, lipid lowering, and anti-inflammatory action. ${ }^{22)}$ Lactucin, 8-deoxylacturin, and lactucopicrin contribute to the bitter taste of lettuce or chicory. Lactucin and lactucopicrin have also been reported as the main active sedative substances in a spontaneous locomotor activity test. ${ }^{20)}$

Green romaine lettuce extract (GRE) was shown to have sleep potentiation activity in a pentobarbital-induced mice sleep model (Fig. 2). Sleep-promoting effects of lettuce were reported in several studies. Ghorbani et al. ${ }^{23)}$ reported that Lactuca sativum hydro-alcoholic extract did not affect sleep latency in pentobarbital-induced mice, but did have a sleepprolonging effect. In addition, our previous studies have confirmed the sleep-promoting effect of green lettuce and we have found that the leaves and seeds of green romaine lettuce were superior to the red lettuce leaves in a pentobarbitalinduced sleep test in mice. ${ }^{21)}$ GRE may reduce the required use of hypnotic drugs to fall asleep in individuals with sleep 
A. NREM

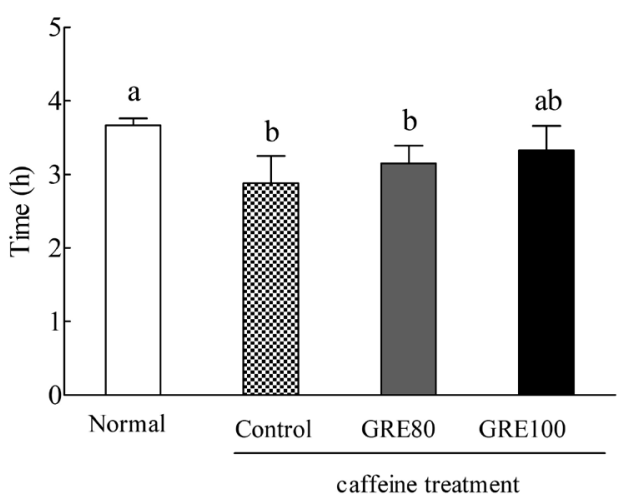

C. Sleep

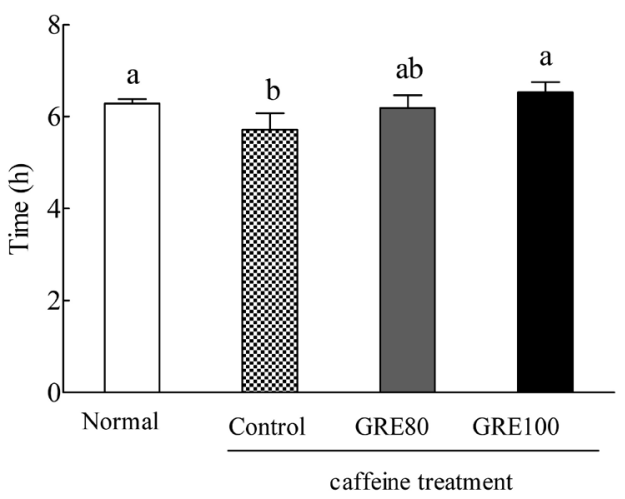

B. REM

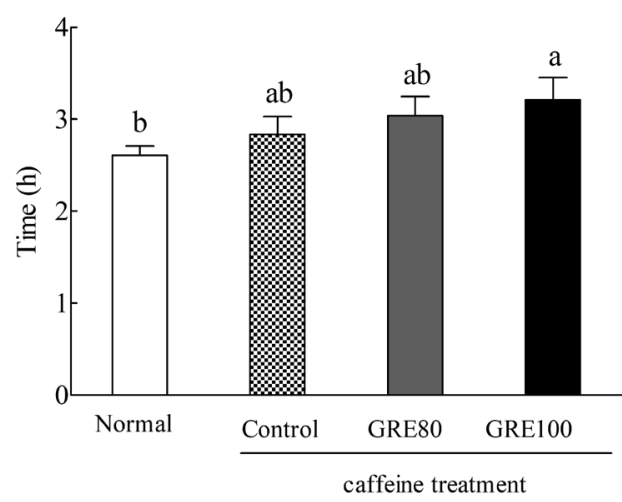

D. Awake

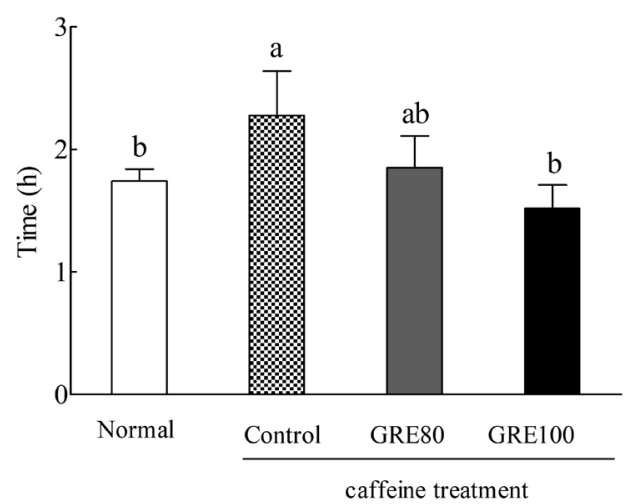

Fig. 4. Effects of Administration of Green Romaine Lettuce Leaf Extract (GRE) on Sleep Quality and Quantity in Caffeine-Induced Wakefulness Rats (Caffeine Dosage of $15 \mathrm{mg} / \mathrm{kg}$ )

Values are the mean \pm S.E.M. calculated from 6 rats. The normal group was administered only $0.9 \%$ physiological saline and the control group was treated with $15 \mathrm{mg} / \mathrm{kg}$ caffeine. Different letters indicate significant differences $(p<0.05)$ among samples by one-way ANOVA followed by post-hoc Tukey test.

A

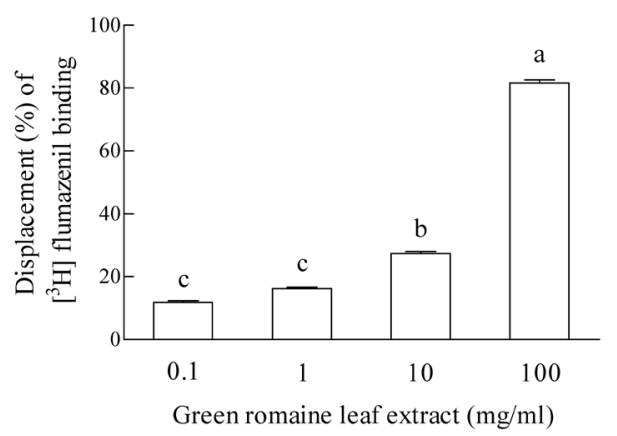

B

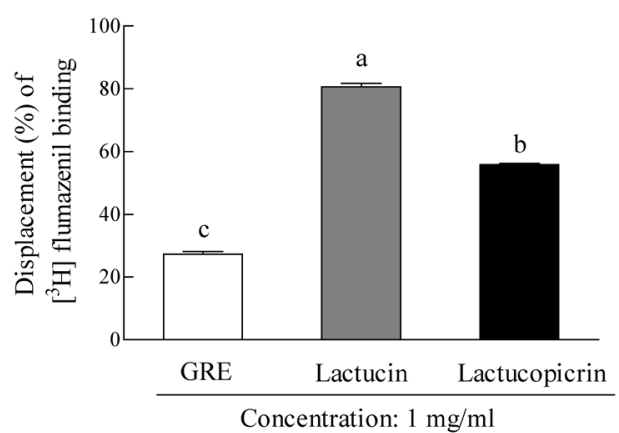

Fig. 5. Displacement of ${ }^{3} \mathrm{H}-$ Flumazenil Binding of Green Romaine Lettuce Leaf Extract (A), Lactucin and Lactucopicrin (B) in the GABA ${ }_{\mathrm{A}} \mathrm{BDZ}$ Receptor Binding Assay

Each data point is expressed as mean \pm S.E.M. for each group, $n=3$. Different letters indicate significant differences $(p<0.05)$ among samples by one-way ANOVA followed by post-hoc Tukey test.

disorders. This supportive effect of GRE on pentobarbital may contribute to ameliorating the adverse effects derived from an overdose of hypnotic drugs.

Therefore, based on the results of GRE increasing the amount of sleep, the sleep structure was investigated through EEG analysis to evaluate whether GRE could improve sleep quality. As a result, delta waves of NREM and total sleep time were increased by administration of GRE (Fig. 3). The two main sleep types are REM (rapid eye movement) and NREM (non-rapid eye movement) sleep. REM sleep is characterized by a decrease in muscle activity and cardiac and respiratory rates, with an increase in brain activity, such as active dreams. Sleep begins with NREM sleep and the NREM-REM sleep cycle repeats 4-5 times at $90-120 \mathrm{~min}$ intervals per night. NREM, characterized by slow brain waves, is reported to occur in deep sleep, while REM has been described as an active form of shallow sleep. ${ }^{24)}$ The NREM consists of three stages, the first stage is the theta wave, and is in a comfort- 

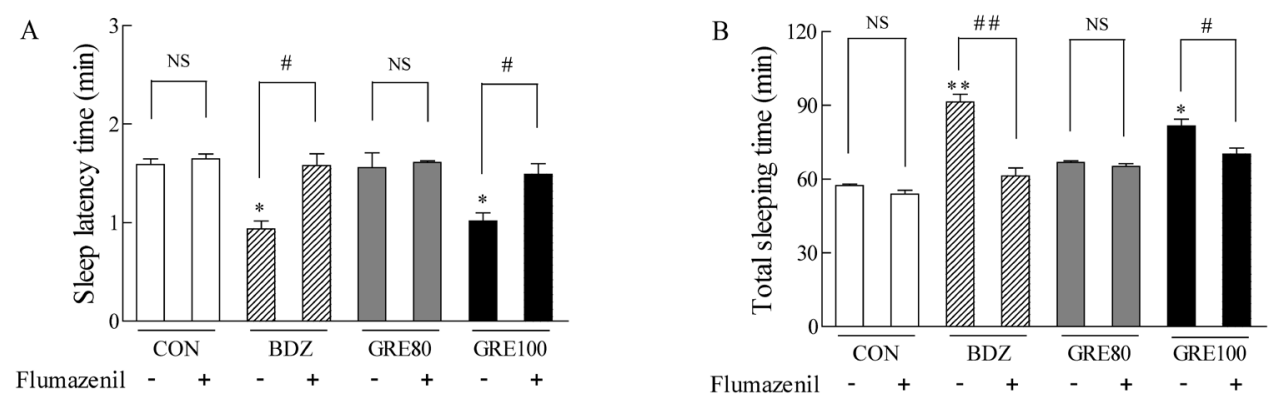

Fig. 6. Effects of Flumazenil on Sleep Latency Time (A) and Total Sleeping Time (B) in Mice Treated with Benzodiazepine (BDZ) or Green Romaine Lettuce Leaf Extract (GRE) at a Hypnotic Dosage of Pentobarbital $(42 \mathrm{mg} / \mathrm{kg}$, i.p.)

Values are presented as means \pm S.E.M. for each group. Symbols indicate significant differences according to two-way ANOVA followed by post-hoc Tukey test: versus the control group at $* p<0.05$ and ${ }^{* *} p<0.01 ;$ versus the treated without flumazenil group at ${ }^{\#} p<0.05$ and ${ }^{\#} p<0.01$. NS, not significant.

able wake state. Eye movements occur in second stages, and the third stage is characterized by slow delta waves. ${ }^{7)}$ In particular, the body is known to restore tissue during slow sleep, where delta waves are generated, and to strengthen the immune system. ${ }^{7)}$ Therefore, we have found that GRE can promote change in healthy sleep architecture.

GRE also exerted a sleep-promoting effect during caffeineinduced insomnia (Fig. 4). Caffeine is a widely used vegetable alkaloid known as a stimulant. ${ }^{25)}$ Caffeine is a methylxanthine compound that increases alertness, induces cortical activation, and reduces fatigue. It blocks the action of the adenosine receptors in the basal forebrain, inducing arousal and reducing slow-wave sleep activity in the cortex. ${ }^{26)}$ Thus, caffeine administration has been used to induce insomnia models in rats. $^{27,28)}$ Our data show that GRE can recover sleep behaviors that are negatively regulated by caffeine (Fig. 4). GRE may alleviate adverse effects of caffeine derived from coffee beverages and drugs that disturb normal sleep.

The ability to modulate $\mathrm{GABA}_{\mathrm{A}}$ receptors has been studied to explore molecular mechanisms underlying these traditional sleep remedies. $\mathrm{GABA}_{\mathrm{A}}$ receptors are a viable target when searching for natural anxiolytic or sedative components from plants. Research has indicated that many herbal extracts are ligands of the $\mathrm{GABA}_{\mathrm{A}}$ receptors in the central nervous system (CNS) and bind to the benzodiazepine binding site, resulting in sleep modulating actions. ${ }^{15,29)}$ Flumazenil, known as an antagonist of benzodiazepine, is a water-soluble imidazobenzodiazephine that acts antagonistically with benzodiazepine in the gamma-amino-butyric acid type $\mathrm{A}\left(\mathrm{GABA}_{\mathrm{A}}\right)$ receptors in the CNS. ${ }^{30)}$ Therefore, by treating with the ${ }^{3} \mathrm{H}$-labeled flumazenil, we investigated whether $\mathrm{GABA}_{\mathrm{A}}-\mathrm{BDZ}$ receptor response is involved in a GRE-mediated sleep promoting effects, and as a result demonstrate that GRE effectively binds to $\mathrm{GABA}_{\mathrm{A}}$ receptors to promote sleep time (Fig. 5A). Green romaine lettuce is expected to contain various active compounds that can affect sleep modulating actions. In particular, lactucin showed a high binding affinity $(80.7 \pm 2.3 \%)$ of the $\mathrm{GABA}_{\mathrm{A}}$-BDZ receptor, and lactucopicrin also binds to the $\mathrm{GABA}_{\mathrm{A}}-\mathrm{BDZ}$ receptor $(55.9 \pm 0.7 \%)$. These results suggest that lactucin and lactucopicrin contained in GRE promotes sleep via GABAergic mechanism (Fig. 5B). In addition, in order to confirm this mechanism in vivo, the effect of flumazenil on the sleep activity of GRE in the pentobarbital-induced sleep model was evaluated. As a result, the sleep enhancement of GRE was completely inhibited by the flumazenil, a specific antagonist of $\mathrm{GABA}_{\mathrm{A}}$-BDZ receptor (Fig. 6). Therefore, in future study, iso- lation of active compounds from green romaine lettuce should provide important information such as the mode of action of romaine lettuce.

Therefore, the results of this study showed that green romaine lettuce contained 1071.7 and $199.2 \mu \mathrm{g} / \mathrm{g}$ of lactucin and lactucopicrin extract, respectively. GRE effectively reduced sleep latency and increased sleep time in a pentobarbitalinduced sleep model. GRE, in particular, increased sleep time by enhancing NREM in sleep architecture. This GREmediated increase of NREM and sleep time was also observed in a caffeine-induced wakefulness model. In a receptor binding assay, the interaction of GRE with GABA receptors was indicated as the primary mechanism by which these sleeppromoting effects occur in rodent models. Taken together, our findings describe that lactucin and lactucopicrin from green romaine lettuce extract are attributed to the quality and quantity of sleep and this result suggests that green romaine leaf extract could be used as sleeping agent.

Acknowledgments This research was supported by 2018 Ottogi foundation research program, Korea (R1727672).

Conflict of Interest The authors declare no conflict of interest.

\section{REFERENCES}

1) Sayyah M, Hadidi N, Kamalinejad M. Analgesic and anti-inflammatory activity of Lactuca sativa seed extract in rats. J. Ethnopharmacol., 92, 325-329 (2004).

2) Slavin JL. Carbohydrates, dietary fiber, and resistant starch in white vegetables: links to health outcomes. Adv. Nutr., 4, 351S-355S (2013).

3) Harsha SN, Anilakumar KR. Anxiolytic property of Lactuca sativa, effect on anxiety behaviour induced by novel food and height. Asian Pac. J. Trop. Med., 6, 532-536 (2013).

4) Harsha S, Anilakumar K. Effects of Lactuca sativa extract on exploratory behavior pattern, locomotor activity and anxiety in mice. Asian Pac. J. Trop. Dis., 2, S475-S479 (2012).

5) Ismail H, Dilshad E, Waheed MT, Mirza B. Transformation of lettuce with rol $\mathrm{ABC}$ genes: extracts show enhanced antioxidant, analgesic, anti-inflammatory, antidepressant, and anticoagulant activities in rats. Appl. Biochem. Biotechnol., 181, 1179-1198 (2017).

6) Siegel JM. Clues to the functions of mammalian sleep. Nature, 437 , 1264-1271 (2005).

7) McKenna JT, Zielinski MR, McCarley RW. Neurobiology of REM sleep, NREM sleep homeostasis, and gamma band oscillations. Sleep Disorders Medicine. (Chokroverty S ed.), Springer, New 
York, pp. 55-77 (2017).

8) Khazipov R, Luhmann HJ. Early patterns of electrical activity in the developing cerebral cortex of humans and rodents. Trends $\mathrm{Neu}$ rosci., 29, 414-418 (2006).

9) Sivertsen B, Krokstad S, Overland S, Mykletun A. The epidemiology of insomnia: associations with physical and mental health: the HUNT-2 study. J. Psychosom. Res., 67, 109-116 (2009).

10) Kato M, Phillips BG, Sigurdsson G, Narkiewicz K, Pesek CA, Somers VK. Effects of sleep deprivation on neural circulatory control. Hypertension, 35, 1173-1175 (2000).

11) Fang XS, Hao JF, Zhou HY, Zhu LX, Wang JH, Song FQ. Pharmacological studies on the sedative-hypnotic effect of semen Ziziphi spinosae (suanzaoren) and radix et rhizoma Salviae miltiorrhizae (danshen) extracts and the synergistic effect of their combinations. Phytomedicine, 17, 75-80 (2010).

12) Abourashed EA, Koetter U, Brattstrom A. In vitro binding experiments with a valerian, hops and their fixed combination extract (Ze91019) to selected central nervous system receptors. Phytomedicine, 11, 633-638 (2004).

13) Srivastava JK, Shankar E, Gupta S. Chamomile: a herbal medicine of the past with a bright future. Mol. Med. Rep., 3, 895-901 (2010).

14) Villet S, Vacher V, Colas A, Danno K, Masson JL, Marijnen P, Bordet MF. Open-label observational study of the homeopathic medicine passiflora compose for anxiety and sleep disorders. Homeopathy, 105, 84-91 (2016).

15) Jo K, Suh HJ, Choi HS. Polygonatum sibiricum rhizome promotes sleep by regulating non-rapid eye movement and GABAergic/serotonergic receptors in rodent models. Biomed. Pharmacother., 105, 167-175 (2018).

16) Hong KB, Han SH, Park Y, Suh HJ, Choi HS. Romaine lettuce/ skullcap mixture improves sleep behavior in vertebrate models. Biol. Pharm. Bull., 41, 1269-1276 (2018).

17) Abu-Reidah IM, Arráez-Román $D$, Quirantes-Piné R, FernándezArroyo S, Segura-Carretero A, Fernández-Gutiérrez A. HPLCESI-Q-TOF-MS for a comprehensive characterization of bioactive phenolic compounds in cucumber whole fruit extract. Food Res. Int., 46, 108-117 (2012).

18) Zhao X, Cui XY, Wang LE, Zhang YH. Potentiating effect of diltiazem on pentobarbital-induced hypnosis is augmented by serotonergic system: The TMN and VLPO as key elements in the pathway.
Neuropharmacology, 56, 937-943 (2009).

19) Risa J, Risa A, Adsersen A, Gauguin B, Stafford GI, van Staden J, Jager AK. Screening of plants used in southern Africa for epilepsy and convulsions in the GABA(A)-benzodiazepine receptor assay. $J$. Ethnopharmacol., 93, 177-182 (2004).

20) Wesotowska A, Nikiforuk A, Michalska K, Kisiel W, ChojnackaWojcik E. Analgesic and sedative activities of lactucin and some lactucin-like guaianolides in mice. J. Ethnopharmacol., 107, 254258 (2006).

21) Kim HD, Hong KB, Noh DO, Suh HJ. Sleep-inducing effect of lettuce (Lactuca sativa) varieties on pentobarbital-induced sleep. Food Sci. Biotechnol., 26, 807-814 (2017).

22) Gromek D, Kisiel W, Klodzińska A, Chojnacka-Wójcik E. Biologically active preparations from Lactuca virosa L. Phytother. Res., 6 , 285-287 (1992)

23) Ghorbani A, Rakhshandeh H, Sadeghnia HR. Potentiating effects of Lactuca sativa on pentobarbital-induced sleep. Iran. J. Pharm. Res., 12, 401-406 (2013).

24) Busek P, Vankova J, Opavsky J, Salinger J, Nevsimalova S. Spectral analysis of the heart rate variability in sleep. Physiol. Res., 54, 369-376 (2005)

25) Jang H-S, Jung JY, Jang I-S, Jang K-H, Kim S-H, Ha J-H, Suk K, Lee M-G. L-Theanine partially counteracts caffeine-induced sleep disturbances in rats. Pharmacol. Biochem. Behav., 101, 217-221 (2012).

26) Nehlig A, Daval JL, Debry G. Caffeine and the central nervous system: mechanisms of action, biochemical, metabolic and psychostimulant effects. Brain Res. Brain Res. Rev., 17, 139-170 (1992).

27) Paterson LM, Wilson SJ, Nutt DJ, Hutson PH, Ivarsson M. A translational, caffeine-induced model of onset insomnia in rats and healthy volunteers. Psychopharmacology, 191, 943-950 (2007).

28) Bonnet MH, Arand D. Caffeine use as a model of acute and chronic insomnia. Sleep, 15, 526-536 (1992).

29) Choi HS, Hong KB, Han SH, Suh HJ. Valerian/cascade mixture promotes sleep by increasing non-rapid eye movement (NREM) in rodent model. Biomed. Pharmacother., 99, 913-920 (2018).

30) Curran HV, Birch B. Differentiating the sedative, psychomotor and amnesic effects of benzodiazepines: a study with midazolam and the benzodiazepine antagonist, flumazenil. Psychopharmacology, 103, 519-523 (1991). 